\title{
Vida y productividad de la herramienta de corte en el fresado de acabado del acero endurecido AISI D6
}

\section{Life and productivity of the cutting tool in milling hardened steel finish AISI D6}

\author{
Yanier Sánchez Hechavarría ${ }^{1} \quad$ Anselmo Eduardo Diniz ${ }^{2} \quad$ Mariza Mariño Cala $^{3}$ \\ Recibido 14 de diciembre de 2015, aceptado 17 de junio de 2016 \\ Received: December 14, 2015 Accepted: June 17, 2016
}

\begin{abstract}
RESUMEN
El fresado de acabado de aceros endurecidos con altas velocidades ha sido limitado por el desgaste prematuro de la herramienta y la inestabilidad dinámica. Durante el fresado de acabado de superficies inclinadas con fresas de espiga de punta redonda, ocurren elevadas interrupciones durante el corte y aparecen fenómenos dinámicos diferentes a aquellos que se presentan durante el fresado de superficies planas. En este trabajo se investiga la influencia de la estabilidad dinámica en la eficiencia y la productividad del fresado de acabado de superficies inclinadas de acero herramental endurecido AISI D6 con altas velocidades. Los experimentos fueron realizados en un centro de mecanizado MORI SEIKI SV40 con mando CNC. En los ensayos se emplearon herramientas intercambiables de carburos cementados y dos portaherramientas, una de acero y otro de carburo cementado. La evaluación de la estabilidad dinámica se basó en el análisis de los espectros de frecuencias de las fuerzas de corte radial obtenidos mediante la Transformada Rápida de Fourier (FFT) y de la Raíz Media Cuadrática $(R M S)$ de las señales de fuerza en el dominio del tiempo. Se comprobó que en los procesos más estables, el volumen de viruta removido y el área cortada por la herramienta son mayores, produciéndose un aumento de la productividad del proceso y obteniéndose además un aumento de la vida útil de la herramienta y mejores resultados en el acabado superficial de la pieza.
\end{abstract}

Palabras clave: Fresado, acero endurecido, productividad, rugosidad superficial, estabilidad dinámica.

\begin{abstract}
Finish milling of hardened steels at high speed has been limited by premature tool wear and dynamic instability. During finish milling of inclined surfaces with ball end mill, high interruptions occur during cutting and dynamic phenomena different to those that occur during milling flat surfaces. In this work the influence of dynamic stability in efficiency and productivity of inclined surface finish milling in hardened steel AISI D6 with high speeds, was studied. The experiments were performed on a machining center MORI SEIKI SV40 with CNC control. They were used as well, interchangeable carbide tools and two tool holder, one of steel and the other of carbide. The evaluation of the dynamic stability was based on the analysis of the spectra frequency of cutting radial forces obtained through Fast Fourier Transform (FFT) and Root Main Square (RMS) of the signal in time domain. It was confirmed that in more stable

1 Departamento de Manufactura y Materiales. Facultad de Ingeniería Mecánica. Universidad de Oriente. Avenida de las Américas s/n. Santiago de Cuba, Cuba. E-mail: rysan@uo.edu.cu

2 Departamento de Fabricação. Faculdade de Engenharia Mecânica. Universidade Estadual de Campinas. Rua Mendeleiev, 200. Caixa postal: 6122. CEP: 13083-860. Cidade Universitária Zeferino Vaz. Campinas. São Paulo. Brasil. E-mail: anselmo@fem.unicamp.br

3 Departamento de Manufactura y Materiales. Facultad de Ingeniería Mecánica. Universidad de Oriente. Avenida de las Américas s/n. Santiago de Cuba, Cuba. E-mail: mmarino@uo.edu.cu

* Autor de correspondencia
\end{abstract}


processes, the volume of removed chip and the cut area by the tool are larger, resulting in an increase of the process productivity and obtaining besides an increase of the tool life and better results in the surface finish workpiece.

Keywords: Milling hardened steel, productivity, roughness surfaces, dynamical stability.

\section{INTRODUCCIÓN}

Actualmente una gran cantidad de productos dependen de moldes metálicos para su producción, donde se necesita que el negativo de ese producto sea confeccionado en los componentes funcionales de los moldes. La complejidad de estos, en los que se combinan superficies rectas e inclinadas con curvas complejas, es uno de los factores determinantes en el tiempo de fabricación y el costo de los moldes metálicos [1].

Debido a los avances tecnológicos en el área de fabricación de cavidades en moldes metálicos, herramientas de corte, desarrollo de nuevas máquinas herramienta y sistemas computacionales para proyectos de manufactura asistida por computadoras CAD/CAM, se hace posible el mecanizado de superficies complejas de materiales endurecidos con altos valores de acabado superficial y dimensional. Además de esto, el conocimiento de las condiciones de corte y la selección de la estrategia de mecanizado adecuada pueden reducir de modo significativo los tiempos de manufactura, aumentar la productividad e influir en la calidad superficial de la pieza [2-3].

El fresado con altas velocidades (HSM) es uno de los métodos más importantes en los procesos de fabricación de moldes metálicos de alta complejidad tecnológica. Para cambiar la forma de una pieza de trabajo y obtener una forma geométrica deseada es necesario el uso de máquinas herramienta, herramientas de corte y estrategias de corte apropiadas, con el objetivo de alcanzar la calidad dimensional y superficial requerida [1].

La aplicación cada vez más común de $H S M$ posibilita el fresado de materiales con dureza mayor de $60 \mathrm{HRC}$, partiendo de la pieza ya endurecida e interviniendo en todo el proceso de producción del molde, se puede lograr hasta un $51 \%$ de reducción del tiempo de producción, al eliminar largos procesos de acabado superficial manual que necesita un personal especializado para su realización [4].
El tipo de proceso de fresado y su régimen de aplicación definen el máximo nivel y la máxima amplitud de las vibraciones. En las operaciones de fresado de desbaste lo que determina este nivel es el efecto que las vibraciones ejercen sobre la vida de la herramienta, siempre y cuando la superficie generada sea adecuada para la operación posterior. En operaciones de acabado, este es determinado por la calidad de la superficie y la precisión dimensional exigida [5].

En el fresado con altas velocidades, las vibraciones relativas entre la pieza y la herramienta, que surgen durante la operación, ejercen gran influencia sobre los resultados del proceso. Estas pueden alcanzar niveles inaceptables, deteriorando el acabado de la superficie y comprometiendo la vida de la herramienta, especialmente en situaciones en que existe una inherente falta de rigidez en el sistema.

Estas condiciones son frecuentemente encontradas en el fresado de moldes metálicos, cuyas geometrías requieren la utilización de herramientas de gran longitud y pequeño diámetro para áreas profundas, a fin de evitar colisiones con las superficies adyacentes. El fresado de estos componentes es una tarea difícil, pues las fuerzas de corte periódicas excitan a la pieza y a la herramienta estática y dinámicamente. Las deflexiones estáticas producen errores de forma y los desplazamientos dinámicos perjudican el acabado de la superficie [6].

Durante el proceso de fresado de superficies inclinadas, la excentricidad producida por la rotación de la herramienta induce desplazamientos del borde cortante de la fresa. Estos desplazamientos generan irregularidades en la superficie fresada afectando la rugosidad superficial de la pieza en el plano de trabajo. Los valores de estos desplazamientos son afectados por la longitud en voladizo de la herramienta, las vibraciones forzadas generadas por la frecuencia de entrada de dientes (f) y autoexcitadas, debido a la variación del espesor de la viruta y las fuerzas centrífugas que aumentan con el aumento de la velocidad de rotación del husillo [7]. 
En procesos de fresado con altas velocidades estables de superficies inclinadas se obtienen valores de rugosidad más bajos que en procesos inestables obteniéndose también aumento de la vida de la herramienta y una aumento de la productividad de la operación tecnológica [8].

Según Antonialli, Diniz y Pederiba [9], la tasa de remoción de material $(Q)$ es un índice de productividad del proceso que indica el volumen de virutas removido en la unidad de tiempo $\left(\mathrm{mm}^{3} /\right.$ min) y puede ser fácilmente empleado en el sector industrial para determinar la cantidad de material que se va a remover durante el proceso de manufactura en función de la velocidad de corte y la velocidad de avance. La tasa de remoción de material puede ser calculada mediante la ecuación (1).

$$
Q=1000 \cdot\left[\frac{a_{e} \cdot a_{p} \cdot V_{c} \cdot s_{z} \cdot z}{\pi \cdot D_{e \max }}\right]
$$

Donde:

Q es el volumen específico de viruta removida.

$\mathrm{a}_{\mathrm{e}}$ es la profundidad de corte radial.

$a_{p}$ es la profundidad de corte axial.

$\mathrm{V}_{\mathrm{C}}$ es la velocidad de corte.

$\mathrm{s}_{\mathrm{z}}$ es el avance por dientes

$z$ es el número de dientes

$\mathrm{D}_{\mathrm{emax}}$ es el diámetro efectivo máximo.

Estos autores determinaron, además, como índices de productividad el área cortada por la herramienta $\left(\mathrm{A}_{\mathrm{C}}\right)$ y el volumen de viruta removido $(\mathrm{V})$. Esta última variable está relacionada directamente con la tasa de remoción de material y la vida útil de la herramienta (T) y puede ser calculada mediante la ecuación (2).

$$
V=Q \cdot T
$$

El presente trabajo tiene como objetivo investigar la influencia de la estabilidad dinámica en la vida y la productividad de la herramienta de corte utilizada en el fresado de acabado de superficies inclinadas de acero endurecido AISI D6 con altas velocidades.

\section{Estrategia de fresado}

Nicola Zeilmann y Missel [10], determinaron que las mejores estrategias para el mecanizado de una superficie inclinada son las horizontales, al obtener los mejores resultados de los parámetros $\mathrm{Ra}$ y $\mathrm{Rz}$ en el sentido del avance y el incremento lateral durante el fresado de una superficie inclinada a $60^{\circ}$ de acero herramental AISI H13 con una dureza de $52 \mathrm{HRC}$. Estos autores plantean que cuando se fresa con grandes ángulos de inclinación de la superficie los mejores resultados durante el corte se consiguen con la estrategia horizontal descendente lográndose los menores valores de rugosidad superficial Ra y $\mathrm{Rz}$ en el sentido del avance, en detrimento de la rugosidad superficial en la dirección del incremento lateral. Los valores de rugosidad en la dirección del incremento lateral (perpendicular al sentido del avance) aumentan, debido al flujo lateral de las virutas removidas [10].

El diámetro de corte efectivo es mayor para las trayectorias ascendentes que para las descendentes, resultando en una velocidad de corte más elevada durante el fresado con trayectorias ascendentes. La región de contacto de la fresa de punta esférica cambia con relación a la inclinación del plano, pues el diámetro efectivo en la región de corte se altera para las trayectorias ascendentes y descendentes [10].

Según Cosma [11] las mejores estrategias para el fresado de superficies inclinadas son la vertical ascendente y la horizontal ascendente, pero la estrategia vertical ascendente tiene muchos inconvenientes para el fresado de cavidades estrechas y profundas.

El fresado horizontal ascendente es más favorable que el horizontal descendente ya que determina una disminución del parámetro Ra en el sentido del avance y del incremento lateral y niveles más bajos de vibraciones [10-12]. Sin embargo, esta estrategia de corte también es poco usada en la elaboración de moldes con cavidades estrechas o complejas debido a que la herramienta debe comenzar el proceso de corte desde el fondo de la cavidad hacia arriba.

\section{MATERIALES Y MÉTODOS}

Para el proceso de fresado se seleccionó una estrategia horizontal descendente, Figura 1, con la que se obtienen buenos valores de rugosidad en el sentido del avance en detrimento de la rugosidad en el sentido de la alimentación (perpendicular al avance).

Los experimentos fueron realizados en un centro de mecanizado vertical MORI SEIKI SV 40 con 


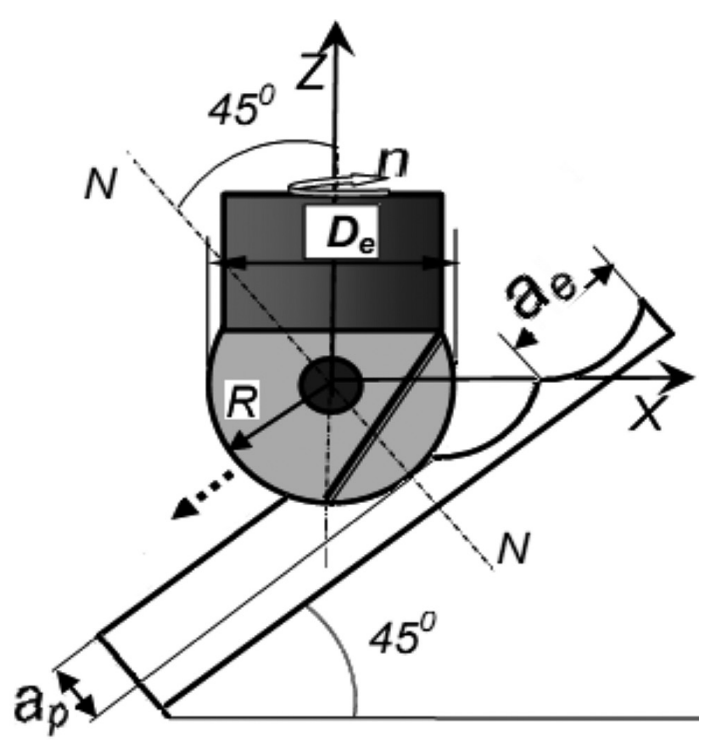

Figura 1. Estrategia de corte horizontal: descendente (modificado) [11].

rotación máxima del husillo de $12.000 \mathrm{rev} / \mathrm{min}$ y potencia principal de $22 \mathrm{~kW}$. El material de los cuerpos de prueba fue fabricado con acero AISI D6 endurecido, el que fue escogido debido a sus aplicaciones en la fabricación de matrices de corte y embutido en frío. La dureza del material utilizado para fabricar el cuerpo de prueba es de $62 \mathrm{HRC}$.

El acero AISI D6 es un acero aleado con alto contenido de carbono y de cromo, con tungsteno en su composición química. Este material tiene una alta tensión de compresión, alta resistencia al desgaste, alta dureza superficial y buena dureza. La composición química y las propiedades mecánicas de este material se muestran en las Tablas 1 y 2.

Para los ensayos se usaron fresas de espiga de punta redonda SANDVIK COROMANT R216F-16 40 E-L de la clase P10A (ISO H10) con recubrimiento TiAlN y diámetro de $16 \mathrm{~mm}$. Para el montaje de

Tabla 1. Composición química del acero herramental AISI D6.

\begin{tabular}{|c|c|c|c|c|c|c|c|}
\hline $\mathbf{C}$ & $\mathbf{M n}$ & $\begin{array}{c}\mathbf{P} \\
\text { máx. }\end{array}$ & $\begin{array}{c}\text { S } \\
\text { máx. }\end{array}$ & $\mathbf{S i}$ & $\mathbf{C r}$ & $\mathbf{W}$ & $\mathbf{V}$ \\
\hline 2,1 & 0,4 & 0,03 & 0,03 & 0,3 & 11,5 & 0,7 & 0,2 \\
\hline
\end{tabular}

Tabla 2. Propiedades mecánicas del acero AISI D6.

\begin{tabular}{|c|c|c|c|}
\hline 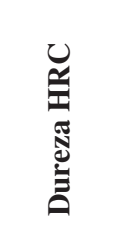 & 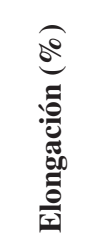 & 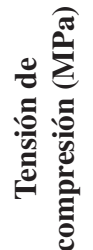 & 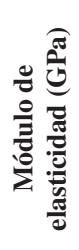 \\
\hline Máx. 62 & $30-32$ & 1320 & 194 \\
\hline
\end{tabular}

las pastillas se usaron dos portaherramientas: uno de acero R216F-16A16S-063 (PH1) y otro de carburos cementados R216F-16A16C-063 (PH2). Los mismos fueron fijados en un mandril hidráulico con una longitud en voladizo de $70 \mathrm{~mm}$. El proceso de corte se realizó en seco.

Las condiciones experimentales usadas en los ensayos se muestran en la Tabla 3.

Cosma [13] concluyó que la forma y el espesor de la viruta varían en función del ángulo de inclinación de la superficie tanto en estrategias de corte descendentes como ascendentes, por lo que se procedió a diseñar la viruta en AUTOCAD 2014 según la metodología establecida por Cosma [14] y luego se procedió a determinar el espesor medio de la viruta para una inclinación de superficie de $45^{\circ}$. Después se realizó un ajuste de las profundidades de corte radial $\left(\mathrm{a}_{\mathrm{e}}\right)$ y axial $\left(\mathrm{a}_{\mathrm{p}}\right)$ para que, con la variación de la inclinación de la superficie a $75^{\circ}$ se mantuviese constante el espesor medio de la viruta $\left(\mathrm{h}_{\mathrm{m}}=0,034 \mathrm{~mm}\right)$ que tiene una

Tabla 3. Condiciones experimentales.

\begin{tabular}{|c|c|c|c|c|c|}
\hline 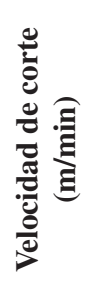 & 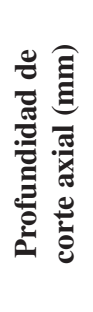 & 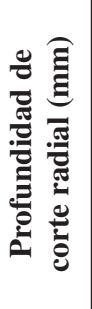 & 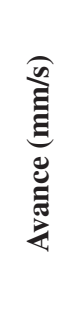 & 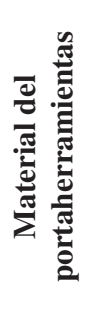 & 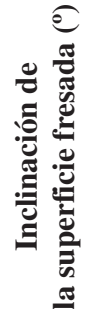 \\
\hline 420 & 0,15 & 0,15 & 0,15 & PH1 & 45 \\
\hline 447,5 & 0,15 & 0,15 & 0,15 & PH1 & 45 \\
\hline 420 & 0,15 & 0,15 & 0,15 & PH2 & 45 \\
\hline 447,5 & 0,15 & 0,15 & 0,15 & PH2 & 45 \\
\hline 420 & 0,175 & 0,15 & 0,15 & PH2 & 75 \\
\hline 447,5 & 0,175 & 0,15 & 0,15 & PH2 & 75 \\
\hline
\end{tabular}


gran influencia sobre las componentes de la fuerza de corte. Se tuvo en cuenta no variar la profundidad de corte radial, ya que tiene gran influencia sobre las componentes tangencial y radial de la fuerza de corte.

Para la selección del criterio de fin de vida de las herramientas se debe tener en cuenta que tipo de operación se realizará. En operaciones de acabado, donde el requisito fundamental es conseguir buen acabado superficial y dimensional además de tolerancias pequeñas, el desgaste de flanco VB no debe exceder de $0,2 \mathrm{~mm}$ para calidades de IT7 y 0,3 $\mathrm{mm}$ para calidades de IT8 [6].

La señal de respuesta en frecuencia del sistema herramienta-portaherramientas-husillo se obtuvo usando un acelerómetro PCB 352C33 (PCB PIEZOTRONICS) en la extremidad de las herramientas, las frecuencias de resonancias de los sistemas fueron excitadas, utilizando un martillo de acero con punta de plástico dotado de un transductor de fuerza piezoeléctrico en las direcciones X e Y.

Para la medición de las fuerzas en las tres direcciones (X, Y, Z) fue fijado un dinamómetro Kistler 9257B en la mesa de máquina, colocándose el cuerpo de prueba sobre la base superior del mismo. Con este tipo de basificación quedan eliminados los grados de libertad de la pieza. El dinamómetro está conectado a un acondicionador de señales Kistler 5019B y la adquisición de los datos se realizó mediante una placa de adquisición de señales A/D National Instruments PCI-6025E, con salida hacia un computador y el empleo del software LabView 8.5 para procesar los resultados.

En el dominio del tiempo fue evaluada la Raíz Media Cuadrática $(R M S)$ de la señal, tomado como parámetro estático a partir de la ecuación (3).

$$
R M S=\sqrt{\frac{1}{N_{P}} \cdot \sum_{i=1}^{N} x_{1}^{2}}
$$

Donde $N_{p}$ es la cantidad de puntos adquiridos y $x_{\mathrm{i}}$ e la variable de la señal.

El análisis de la $R M S$ de las componentes tangencial, radial y axial de la fuerza de corte nos permite determinar la amplitud y magnitud de las señales fuerza en el dominio del tiempo.
El desgaste de flanco $\left(\mathrm{VB}_{\mathrm{B}}\right)$ de las herramientas utilizadas fue monitoreado con el empleo de un estereoscopio marca QUIMIS montado en conjunto con una cámara fotográfica digital MOTICAM 1000 de 1,3 MP (Figura 2), interconectada al programa analizador de imágenes MOTIC IMAGES PLUS 2.0 ML. Inicialmente se realizó la calibración del sistema para correlacionar una unidad de la figura en pixeles con una unidad métrica, en este caso milímetros.

El uso de este sistema permite el monitoreo del desgaste con rapidez y eficacia. Las mediciones se realizaron por cada 100 pasadas de la herramienta sobre la pieza.

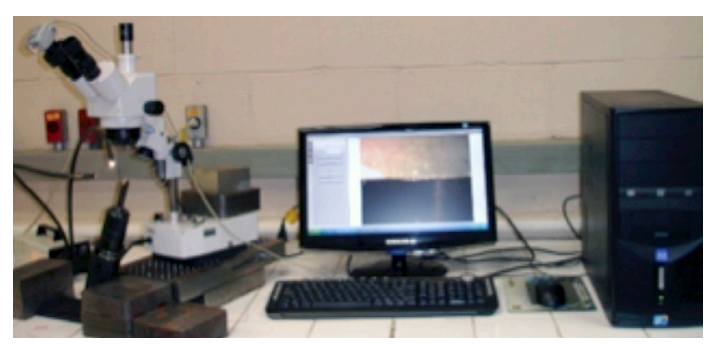

Figura 2. Instalación para la medición del desgaste.

\section{RESULTADOS}

La función de transferencia $\mathrm{H}(\omega)$ de adquisición en los ensayos de análisis de vibraciones se calcula mediante la ecuación (4).

$$
H \omega=\frac{X \omega}{F \omega}
$$

Donde $X \omega\left(\mathrm{m} / \mathrm{s}^{2}\right)$ es la magnitud de la frecuencia adquirida por el acelerómetro y $F \omega$ es la fuerza medida por el transductor de fuerza piezoeléctrico con el que está equipado el martillo [9].

Los componentes tangenciales, radiales y axiales de la fuerza de corte en la herramienta, $F_{t}, F_{r}$ y $F_{a}$, respectivamente, son funciones de ángulo instantáneo $(\varphi)$ en la dirección del avance y pueden ser obtenidos mediante la ecuación 5 [9].

$$
\left[\begin{array}{l}
F_{t} \\
F_{r} \\
F_{a}
\end{array}\right]=\left[\begin{array}{ccc}
\operatorname{sen} \varphi & \cos \varphi & 0 \\
\cos \varphi & \operatorname{sen} \varphi & 0 \\
0 & 0 & 1
\end{array}\right] \cdot\left[\begin{array}{c}
F_{x} \\
F_{y} \\
F_{z}
\end{array}\right]
$$


Las componentes de la fuerza medidos se consideraron en la dirección de los tres ejes de la máquina herramienta $\left(F_{x}, F_{y}\right.$ y $\left.F_{z}\right)$ y a partir de la ecuación (4) se obtuvieron las componentes $F_{t}, F_{r}$ y $F_{a}$.

En las Figuras 3a y 3b se muestran las funciones de transferencia para ambos portaherramientas, de acero y de carburos cementado en el dominio de frecuencia; indicando que la frecuencia natural predominante para ambos portaherramientas son superiores a $1.500 \mathrm{~Hz}$. En estas figuras el gráfico de la función de trasferencia en color azul se corresponden con la dirección X-X y en color negro con la dirección Y-Y.

En determinados rangos de frecuencia de 400 a $1.000 \mathrm{~Hz}$, Figura 4, las funciones de transferencia tienden a aumentar por encima de $0,05 \mathrm{~m} / \mathrm{Ns}^{2}$ donde las herramientas no tendrían la posibilidad de comportarse como cuerpos rígidos si la excitación ocurre en esas frecuencias, por lo que las herramientas serán sometidas a una mayor aceleración si las fuerzas son aplicadas en esta banda de frecuencias [9].
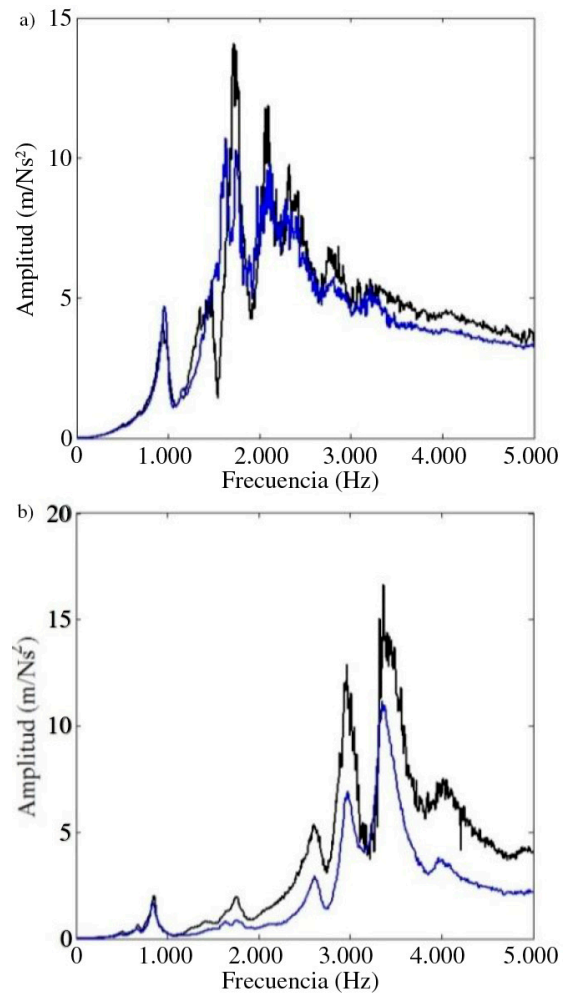

Figura 3. Función de transferencia del portaherramientas: a) R216F-16A16S-063(PH1). b) R216F-16A16C-063 (PH2).



b)

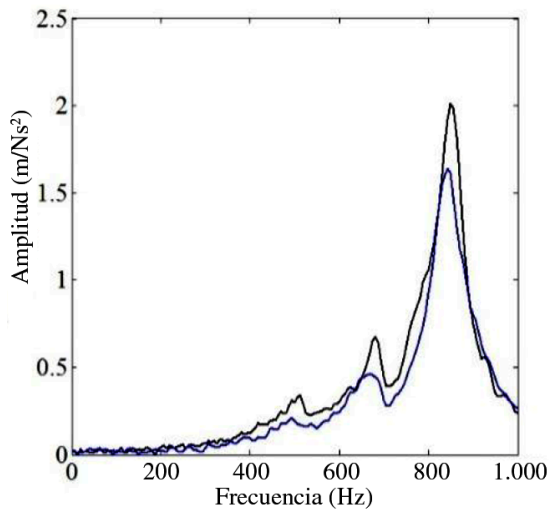

Figura 4. Función de transferencia del portaherramientas: a) R216F-16A16S-063 (PH1), b) R216F-16A16C-063 (PH2). Banda de frecuencia de 0 a $1.000 \mathrm{~Hz}$.

En las señales de fuerza en función de la frecuencia mostradas en la Figura 5 se puede observar en el rango de frecuencias de 400 a $1.000 \mathrm{~Hz}$, las componentes de la fuerza de corte provocan vibraciones forzadas de mayor amplitud. Este comportamiento determina que en cualquier modo estructural la herramienta estará en cierta forma excitada por la frecuencia de entrada de dientes, la frecuencia de rotación del husillo y sus armónicos.

En las Figuras 5a y 5b, $\mathrm{n}$ indica la frecuencia de rotación del husillo y f la frecuencia de entrada de dientes. En condiciones de corte estables, donde no se observan la presencia de vibraciones autoexcitadas, como las mostradas en la Figuras 5a y 5 b, el espectro de frecuencias es dominado por la frecuencia de rotación del husillo y la frecuencia de entrada de dientes y sus armónicos. En esta figura se observa que los picos de frecuencias obtenido con 
el portaherramientas $\mathrm{PH} 1$ (Figura 5a) tienen mayor amplitud que los obtenidos durante el fresado con el portaherramientas $\mathrm{PH} 2$, por lo que las vibraciones forzadas generadas por las componentes de la fuerza de corte serán menores cuando se usa el portaherramientas $\mathrm{PH} 2$.

En la Figura 6 se muestran las señales de fuerza en el dominio del tiempo durante el fresado de superficies inclinadas con iguales valores de $\mathrm{V}_{\mathrm{C}}$ y $\theta$ y diferentes portaherramientas. Las vibraciones generadas por la fuerza de corte tienen mayor amplitud cuando se fresa con el portaherramientas PH1 que cuando se fresa con el portaherramientas $\mathrm{PH} 2$.

Por los resultados obtenidos, se puede plantear que en el proceso de fresado de superficies inclinadas, las vibraciones forzadas generadas por los armónicos de la frecuencia de rotación del husillo y de la frecuencia de entrada de dientes tendrán menor

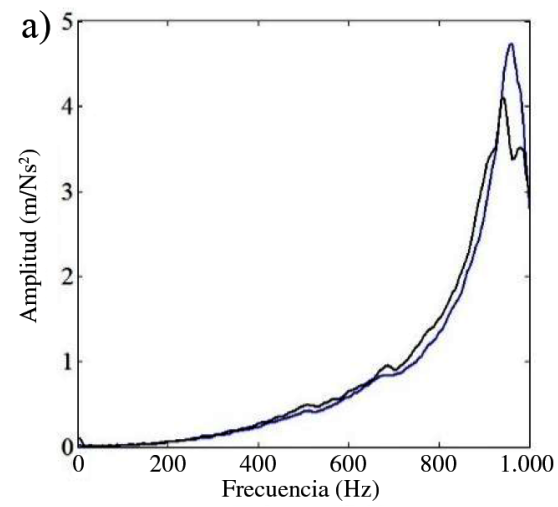

b)

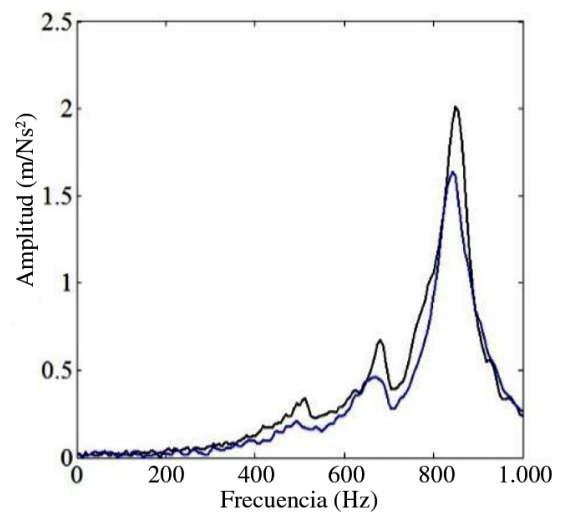

Figura 5. Fuerza radial en el dominio de las frecuencias. $V_{C}=420 \mathrm{~m} / \mathrm{min}, 45^{\circ}$. a) $\mathrm{PH} 1$. b) $\mathrm{PH} 2$. amplitud cuando se usa el portaherramientas $\mathrm{PH} 2$ debido a su mayor rigidez mecánica. Polli [8] obtuvo resultados similares durante el fresado de superficies inclinadas de un acero AISI P20 con 54 HRC de dureza. Esto se cumple siempre y cuando ambos portaherramientas tengan las mismas dimensiones como también fue determinado por [16].

La Figura 7 muestra las $R M S$ de las señales obtenidas para diferentes velocidades de corte y los portaherramientas $\mathrm{PH} 1$ y $\mathrm{PH} 2$ durante el fresado de superficies inclinadas a $45^{\circ}$. Los resultados muestran que las $R M S$ obtenidas durante el fresado con el portaherramientas ( $\mathrm{PH} 2)$ fueron menores que las obtenidas durante el fresado con el portaherramientas (PH1) para los dos valores de velocidades de corte utilizadas en los ensayos. Esto se debe fundamentalmente a que el portaherramientas PH2 tiene mayor módulo de elasticidad y peso (600 GPa y 0,44 kg) que el portaherramientas PH1 (200

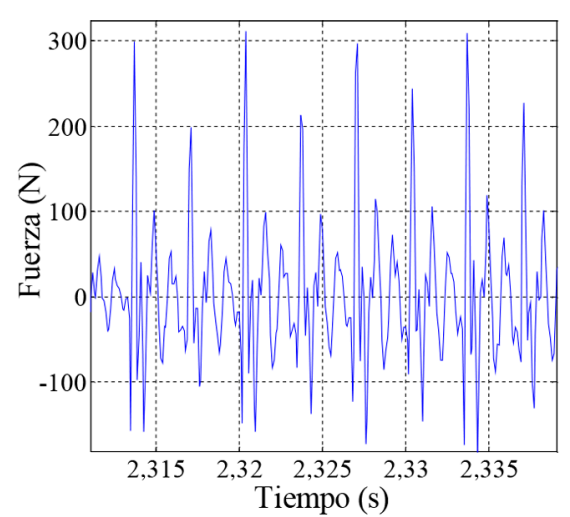

a)

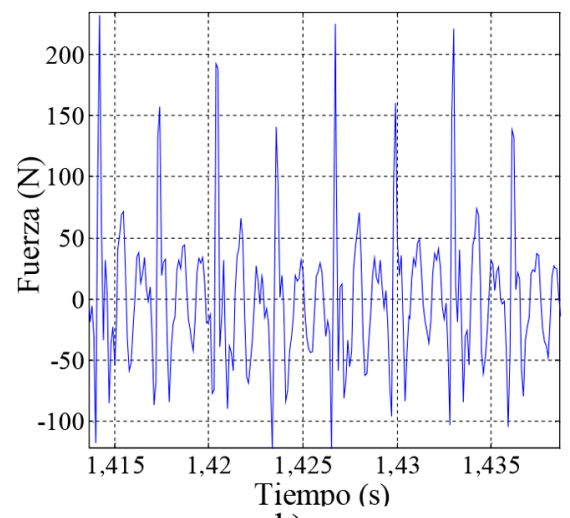

b)

Figura 6. Fuerza radial en el dominio del tiempo. $\mathrm{V}_{\mathrm{C}}=420 \mathrm{~m} / \mathrm{min}, 45^{\circ}$ a). PH1. b) PH2. 


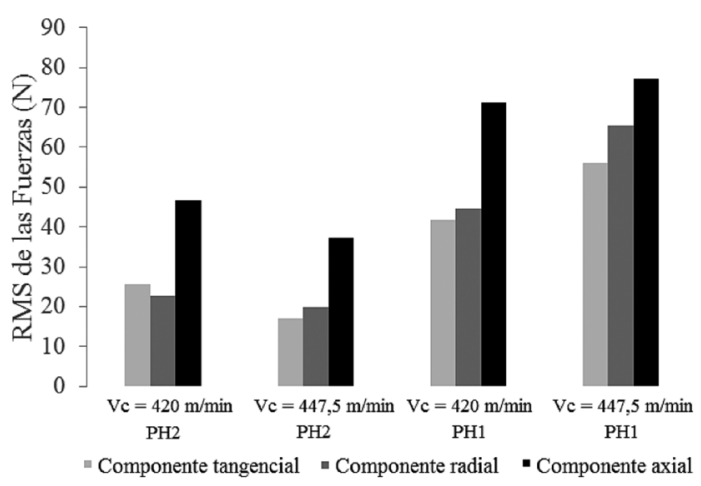

Figura 7. Valores de RMS de los componentes de la fuerza de corte en función del material del portaherramientas.

GPa y $0,305 \mathrm{~kg}$ ), por lo que la deflexión radial del portaherramientas $\mathrm{PH} 2$ será menor que la del portaherramientas $\mathrm{PH} 1$.

En la Figura 8 se muestran las $R M S$ de las señales de fuerza en el dominio del tiempo, obtenidas para diferentes velocidades de corte y ángulos de inclinación de la superficie fresada. En esta figura se observa que la componente axial es mayor durante el fresado de superficies de $45^{\circ}$. En esta dirección la herramienta de corte se comporta como un elemento rígido. Sin embargo, las componentes radiales y tangenciales de la fuerza de corte son menores, durante el fresado de superficies inclinadas a $45^{\circ}$ lo que indica que en estas direcciones se generarán vibraciones

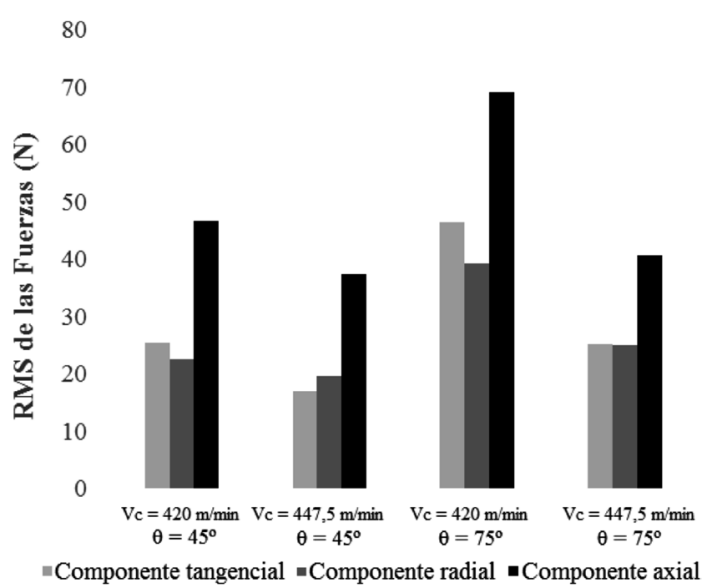

Figura 8. Valores de RMS de los componentes de la fuerza de corte en función del ángulo de inclinación de la superficie. de menor magnitud que durante el fresado de superficies inclinadas a $75^{\circ}$.

De Oliveira [17], demostró que con una inclinación de la superficie fresada de $45^{\circ}$, las componentes de la fuerza de corte en el sentido radial y axial tienen el mismo módulo; pero con una inclinación de $75^{\circ}$, la componente en el sentido radial es mayor que en el sentido axial. Este autor realizó un análisis de varianza que demostró que la inclinación de la superficie a fresar tiene una influencia significativa en la vida de la herramienta con un intervalo de confianza de $99 \%$.

En la Figura 9 se muestra la señal de la componente radial de la fuerza de corte en el dominio de la frecuencia, obtenida durante el fresado de una superficie inclinada a $75^{\circ} \mathrm{con}$ velocidad de corte igual a $420 \mathrm{~m} / \mathrm{min}$. En la misma se observan vibraciones autoexcitadas, las que producen una disminución de la vida de la herramienta de corte debido a la inestabilidad dinámica del proceso de fresado bajo estas condiciones como se observa en la Figura 10.

Influencia de la inclinación de la superficie mecanizada en la vida de la herramienta

Para determinar el punto en el cual el desgaste coincide con el criterio de fin de vida seleccionado $\left(\mathrm{VB}_{\text {máx }}=0,2 \mathrm{~mm}\right)$, se obtuvieron las ecuaciones paramétricas de las curvas de desgaste de flanco en función del tiempo con una significancia de $99 \%$

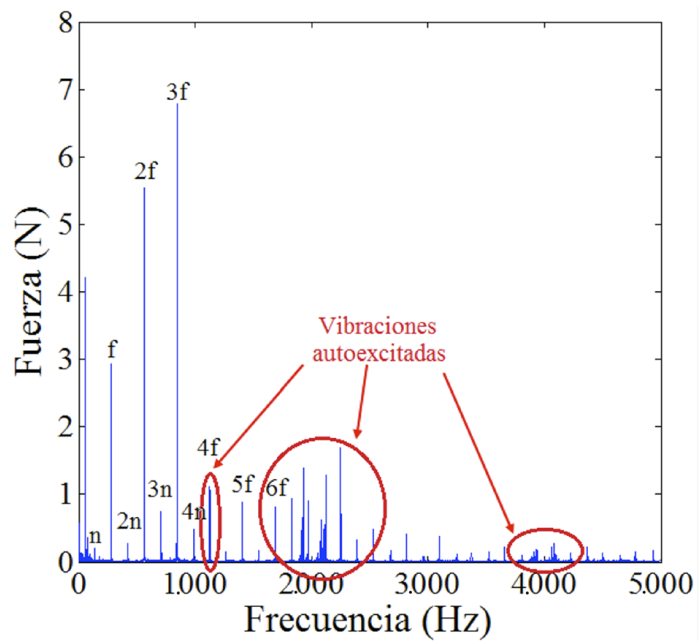

Figura 9. Fuerza radial en el dominio de las frecuencias tiempo. $\theta=75^{\circ}, \mathrm{V}_{\mathrm{C}}=420$ $\mathrm{m} / \mathrm{min}, \mathrm{VB}=0,191 \mathrm{~mm}$. 


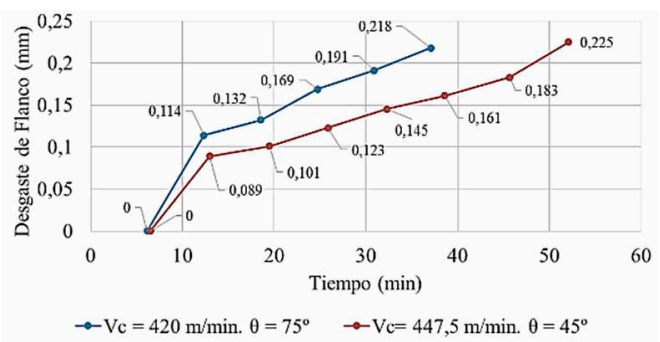

Figura 10. Curva de desgaste. $\mathrm{V}_{\mathrm{C}}=420 \mathrm{~m} / \mathrm{min}$ y $\theta$ $=75^{\circ}$. $\mathrm{V}_{\mathrm{C}}=447,5 \mathrm{~m} / \mathrm{min}$ y $\theta=45^{\circ}$.

utilizando el software STATISTICA 10, versión 10.0.228.2.

En los resultados obtenidos en las curvas de desgaste mostradas en las Figuras 10 y 11, se aprecia que el mejor resultado en cuanto a vida útil de la herramienta se obtuvo cuando se realiza el fresado de la superficie inclinada a $45^{\circ}$ con velocidad de corte igual a 447,5 $\mathrm{m} / \mathrm{min}$. Para esta condición experimental el valor de las $R M S$ de las componentes radiales y tangenciales de la fuerza de corte obtenida al final de la vida de la herramienta fue menor a pesar del aumento de la velocidad de rotación del husillo y por tanto de la frecuencia de entrada de dientes (Figura 8). Este fenómeno se debe a que el tercer armónico de la frecuencia de entrada de dientes $(3 \mathrm{f}=958,3 \mathrm{~Hz})$ está más alejado de la frecuencia modal $1\left(\mathrm{f}_{\mathrm{M} 1}=\right.$ $841 \mathrm{~Hz}$ ) como se muestra en la Tabla 4.

La menor vida útil de la herramienta de corte se obtuvo durante el fresado de la superficie inclinada a $75^{\circ}$ con una velocidad de corte de $420 \mathrm{~m} / \mathrm{min}$ (Figura 10), debido a que $3 \mathrm{f}(843 \mathrm{~Hz})$ para esta condición experimental está muy cercano a $\mathrm{f}_{\mathrm{M} 1}$ $(841 \mathrm{~Hz})$, generándose de esta forma una mayor inestabilidad dinámica en el proceso debido al aumento de las vibraciones forzadas producidas por el aumento del valor modular de las componentes radiales y tangenciales de la fuerza de corte y las $R M S$ de estas componentes, como se aprecia en las Figuras 6 y 8.

En la Tabla 4 se muestran las frecuencias de entrada de dientes (f) y los armónicos más cercanos a la frecuencia modal 1.

Los resultados mostrados en la Figura 11 muestran una pequeña variación en la vida de la herramienta para estas condiciones experimentales $\left(\mathrm{V}_{\mathrm{C}}=420\right.$
Tabla 4. Frecuencia de entrada de dientes y armónicos cercanos a la frecuencia modal 1.

\begin{tabular}{|c|c|c|c|c|}
\hline 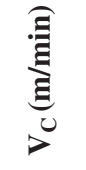 & $\underset{0}{\mathfrak{e}}$ & $\underbrace{\stackrel{⿴ 囗 玉}{\Xi}}_{\mathfrak{\Xi}}$ & $\underset{\mathbb{N}}{\overparen{Z}}$ & 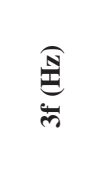 \\
\hline 420 & 45 & 8.995 & 299,8 & 899,4 \\
\hline 447,5 & 45 & 9.583 & 319,4 & 958,3 \\
\hline 420 & 75 & 8.443 & 281,6 & 844,8 \\
\hline 447,5 & 75 & 8.995 & 299,8 & 899,4 \\
\hline
\end{tabular}

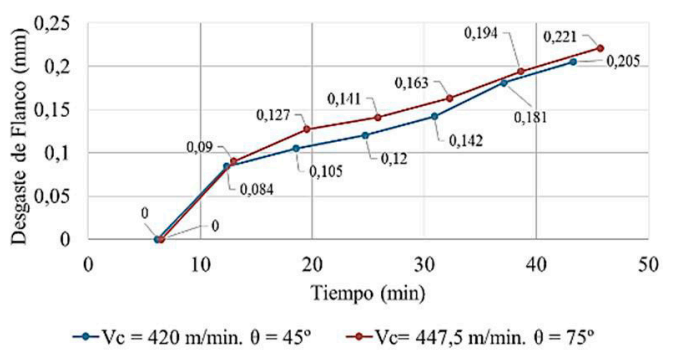

Figura 11. Curva de desgaste. $\mathrm{V}_{\mathrm{C}}=447,5 \mathrm{~m} / \mathrm{min} \mathrm{y}$ $\theta=75^{\circ}$. $V_{C}=420 \mathrm{~m} / \mathrm{min}$ y $\theta=45^{\circ}$.

$\mathrm{m} / \mathrm{min}$ y $\left.\theta=45^{\circ}\right)$ y $\left(\mathrm{V}_{\mathrm{C}}=447,5 \mathrm{~m} / \mathrm{min}\right.$ y $\left.\theta=75^{\circ}\right)$ fresando con el portaherramientas $\mathrm{PH} 2$. Este resultado se debe a que la frecuencia de entrada de dientes y sus armónicos (Tabla 4), se generan en el mismo valor de frecuencias, aun cuando el $R M S$ de la componente radial obtenida durante el fresado de la superficie con inclinación de $75^{\circ}$ y velocidad de corte de 447,5 $\mathrm{m} / \mathrm{min}$ es mayor.

En la Tabla 5 se muestra vida útil de la herramienta (T) en minutos para cada réplica de los ensayos en función del material del portaherramientas y de la velocidad de corte.

Un aumento del ángulo de inclinación de la superficie fresada de $45^{\circ}$ a $75^{\circ}$ produce una disminución de la vida útil de la herramienta, como se puede apreciar en la Tabla 5. Durante el fresado con velocidad de corte de 447,5 $\mathrm{m} / \mathrm{min}$ se produce una disminución de la vida útil de la herramienta, entre 15,8 a 16,3\%; mientras que durante el fresado con velocidad de corte de $420 \mathrm{~m} / \mathrm{min}$ la disminución de la vida útil de la herramienta varió entre 21,1 a $21,6 \%$.

De lo anterior se puede concluir que el aumento de la inclinación de la superficie mecanizada produce 
Tabla 5. Vida útil de la herramienta (T) en función del ángulo de inclinación de la superficie fresada.

\begin{tabular}{|c|c|c|c|c|}
\cline { 3 - 5 } \multicolumn{2}{c|}{} & \multicolumn{3}{c|}{ T (min) } \\
\hline $\boldsymbol{\theta}\left({ }^{\mathbf{o}}\right)$ & $\mathbf{V}_{\mathbf{C}}(\mathbf{m} / \mathbf{m i n})$ & $\begin{array}{c}\mathbf{1}^{\text {ra }} \\
\text { réplica }^{\text {da }}\end{array}$ & $\begin{array}{c}\mathbf{2}^{\text {da }} \\
\text { réplica }\end{array}$ & $\begin{array}{c}\mathbf{3}^{\text {ra }} \\
\text { réplica }\end{array}$ \\
\hline 45 & 420 & 42,00 & 42,30 & 42,23 \\
\hline 45 & 447,5 & 47,63 & 47,46 & 47,91 \\
\hline 75 & 420 & 33,11 & 33,15 & 33,32 \\
\hline 75 & 447,5 & 40,20 & 39,95 & 40,09 \\
\hline
\end{tabular}

una disminución de la estabilidad dinámica del proceso de fresado debido al aumento de los valores de los componentes radiales y tangenciales de las fuerza de corte, lo que provoca una disminución de la vida útil de la herramienta de corte.

Durante el fresado de la superficie inclinada a $45^{\circ}$ con velocidad de corte de $420 \mathrm{~m} / \mathrm{min}$ la velocidad de rotación del husillo es la misma que durante el fresado de la superficie inclinada a $75^{\circ}$ con velocidad de corte de 447,5 $\mathrm{m} / \mathrm{min}$, por lo que la frecuencia de entrada de dientes fue la misma. Para estas condiciones, la disminución de la vida útil de la herramienta con el aumento del ángulo de inclinación de la superficie estuvo entre 4,3\% y 5,6\%.

\section{Influencia del material del portaherramientas en la vida útil de la herramienta}

Analizando los resultados mostrados en la tabla 6 se verifica que el material del portaherramientas tiene mayor influencia sobre la vida útil de la herramienta que la velocidad de corte, los mejores resultados en cuanto a vida de la herramienta se verifican cuando se realiza el fresado usando portaherramientas $\mathrm{PH} 2$ y velocidad de corte de 447,5 m/min. Para esta condición, donde se emplea el portaherramientas de mayor rigidez mecánica, los armónicos de la frecuencia de entrada de dientes, están más alejados de la $\mathrm{f}_{\mathrm{M} 1}$; siendo esta la condición de mayor estabilidad dinámica.

Para $\mathrm{V}_{\mathrm{C}}=447,5 \mathrm{~m} / \mathrm{min}$, la disminución de la vida útil de las herramientas instaladas en el portaherramientas PH1 $\left(\mathrm{f}_{\mathrm{M} 1}=956 \mathrm{~Hz}\right)$ con respecto a las instaladas en el portaherramientas $\mathrm{PH} 2\left(\mathrm{f}_{\mathrm{M} 1}=841 \mathrm{~Hz}\right)$ estuvo entre $35,6 \%$ y $38,6 \%$. Para ambos portaherramientas el tercer armónico de la frecuencia de entrada de dientes, $3 \mathrm{f}=958,3 \mathrm{~Hz}$, que está más próximo de la frecuencia modal del portaherramientas $\mathrm{PH} 1$, por lo que las vibraciones forzadas producidas por la frecuencia de entrada de dientes y sus armónicos serán mayores cuando se usa este portaherramientas.

Tabla 6. Vida útil de la herramienta (T) en función del material del portaherramientas.

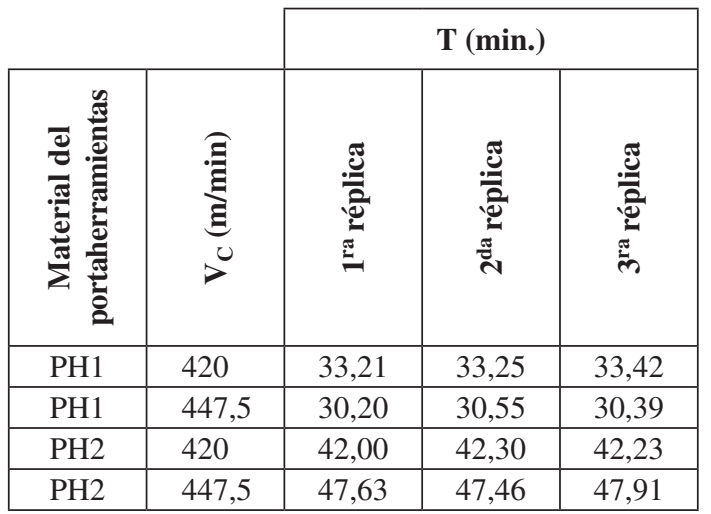

Resultados similares se obtuvieron para velocidad de corte de $420 \mathrm{~m} / \mathrm{min}$, donde las herramientas de instaladas en el portaherramientas $\mathrm{PH} 1$ tuvieron una disminución de la vida entre $20,9 \%$ y $21,5 \%$ con respecto a las herramientas instaladas en el portaherramientas $\mathrm{PH} 2$. Los resultados obtenidos para el portaherramientas $\mathrm{PH} 2$, señalan que el aumento de la estabilidad dinámica provoca un aumento de la vida media de la herramienta en un $11,5 \%$.

En las Figuras 12 y 13 se observan las curvas de desgaste de las herramientas obtenidas para la segunda réplica de los ensayos. En la misma se observa que las herramientas instaladas en el portaherramientas $\mathrm{PH} 2$ comenzaron a desgastarse después que las instaladas en el portaherramientas $\mathrm{PH} 1$, lo demuestra que en procesos dinámicamente más estables es posible aumentar la velocidad de corte y de avance y, por ende, aumentar el área cortada por la herramienta y el volumen de removido, disminuyendo los costos de herramental. Estos resultados coinciden con los obtenidos por De Oliveira [16] durante el fresado de los aceros herramentales endurecidos AISI H13 y AISI D2 con durezas de 52 y, 54 HRC de dureza, utilizando herramientas similares.

Polli [8] observó un comportamiento semejante al mostrado en las Figuras 10 y 11 durante el fresado de acabado de superficies inclinadas de 


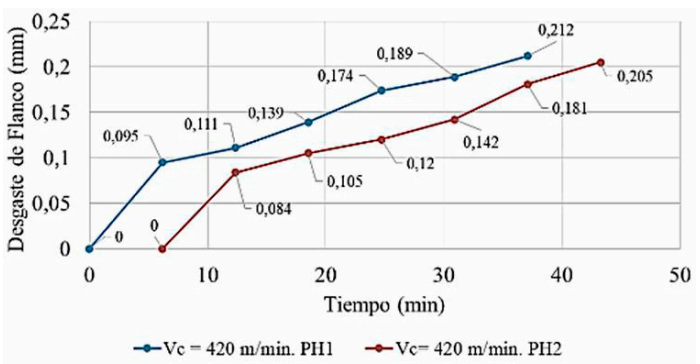

Figura 12. Curva de desgaste de los portaherramientas PH1 y PH2. $\mathrm{V}_{\mathrm{C}}=420 \mathrm{~m} / \mathrm{min}$.

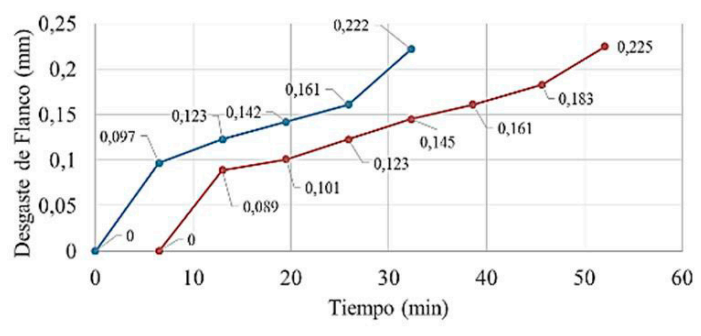

$\rightarrow \mathrm{Vc}=447,5 \mathrm{~m} / \mathrm{min}$. PHI $\rightarrow \mathrm{Vc}=447,5 \mathrm{~m} / \mathrm{min}$. $\mathrm{PH} 2$

Figura 13. Curva de desgaste de los portaherramientas PH1 y PH2. $V_{C}=447,5 \mathrm{~m} / \mathrm{min}$.

acero endurecido AISI H13, se obtienen menores valores de rugosidad superficial al realizar ensayos con portaherramientas de carburos cementados no intercambiables, y portaherramientas de metal duro y acero con calzos intercambiables durante el fresado superficies con ángulos de inclinación de $15^{\circ}$ y $45^{\circ}$.

De los resultados mostrados en la Figura 14 se puede concluir, en los procesos de fresado donde se usó el portaherramientas $\mathrm{PH} 2$, que son dinámicamente más estables, se obtuvieron los mejores resultados en cuanto a volumen de viruta removido y el área cortada por la herramienta, demostrando de esta forma que la productividad del proceso de fresado de superficies inclinadas depende en mayor cuantía de la estabilidad dinámica. Estos resultados coinciden con los obtenidos por [8-9, 14].

\section{CONCLUSIONES}

El aumento del ángulo de inclinación de la superficie fresada de $45^{\circ}$ a $75^{\circ}$ produce una aumento de las $R M S$ de las componentes radial y tangencial de fuerza de corte, indicando esto, que las vibraciones que se generan durante el fresado de superficies

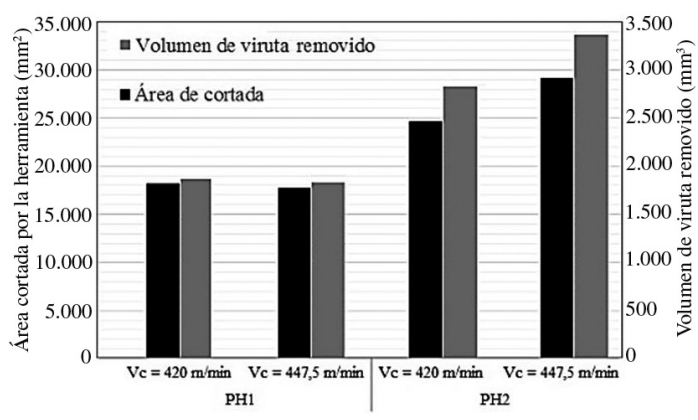

Figura 14. Productividad de la herramienta de corte en función del material del portaherramientas.

inclinadas y, por ende, la inestabilidad dinámica del proceso, aumentan al aumentar la inclinación de la superficie fresada.

Los resultados muestran que las $R M S$ de las componentes radial y tangencial de fuerza de corte, obtenidas durante el fresado de superficies inclinadas a $45^{\circ}$ con el portaherramientas $\mathrm{PH} 2$ fueron menores que las obtenidas durante el fresado con el portaherramientas $\mathrm{PH} 1$ para los valores de velocidades de corte de 420 y 447,5 m/min, por lo que la estabilidad dinámica del proceso de fresado será mayor cuando se usa el portaherramientas $\mathrm{PH} 2$ que tiene mayor módulo de elasticidad.

El mejor resultado en cuanto a vida útil de la herramienta, 47,91 min, se obtuvo durante el fresado de la superficie inclinada a $45^{\circ}$ con el portaherramientas $\mathrm{PH} 2$ y velocidad de corte igual a $447,5 \mathrm{~m} / \mathrm{min}$. Esto se debe a que la componente radial de la fuerza de corte es menor durante el fresado de superficies inclinadas a $45^{\circ}$, lo que indica que en la dirección radial, se generarán vibraciones de menor magnitud y el proceso de fresado es más estable que cuando se realiza el mecanizado de superficies con una inclinación de $75^{\circ}$.

Durante el proceso de fresado de la superficie inclinada a $45^{\circ}$ con el portaherramientas $\mathrm{PH} 2$ y velocidad de corte de $447,5 \mathrm{~m} / \mathrm{min}$, se obtuvieron los mejores resultados en cuanto a volumen de viruta removido y área cortada por la herramienta, demostrando de esta forma que la productividad del proceso de fresado de superficies inclinadas depende en mayor cuantía de la estabilidad dinámica que de la velocidad de corte. 


\section{REFERENCIAS}

[1] A. Gok, C. Gologlu, I.H. Demirci and M. Kurt. "Determination of Surface Qualities on Inclined Surface Machining with Acoustic Sound Pressure". Journal of Mechanical Engineering. Vol. $58 \mathrm{~N}^{\mathrm{o}} 3$, pp. 587-596. 2012. DOI: $10.5545 / \mathrm{sv}-\mathrm{jme}$.2012.352. URL: http://ojs.sv-jme.eu/index.php/sv-jme/article/ view/sv-jme.2012.352

[2] K.T. Ulrich and S. D. Eppinger. "Product Design and Development". $3^{\text {rd }}$ Edition. McGraw-Hill. New York, Estados Unidos. 2004. ISBN-13: 978-0073404776 e ISBN10: 0073404772.

[3] J.E. Shigley, C.R. Mischke and R.G. Budynas. "Mechanical Engineering Design". $3^{\text {rd }}$ Edition. McGraw-Hill. New York, Estados Unidos. 2004. ISBN 0-07-252036-1.

[4] F. Martínez y T. Sánchez. "Conceptos del maquinado con altas velocidades de corte aplicado en moldes y matrices". Ingeniería Mecánica. Vol. 1, pp. 63-65. 2007. ISSN: 1815-5944. URL: http://www.redalyc.org/ articulo.oa?id=225117649009

[5] D. Huo and K. Cheng. "Machining Dynamics. Fundamentals, Applications and Practices". Springer-Verlag London Limited. Londres, Inglaterra. 2009. ISBN: 978-1-84628-367-3 e ISBN: 978-1-84628-368-0.

[6] E. Budak and Y. Altintas. "Peripheral Milling Conditions for Improved Dimensional Accuracy". International Journal of Machine Tools \& Manufacture. Vol. 34 No 7, pp. 907-918. 1994. ISSN: 0890-6955. DOI: 10.1016/0890-6955(94)90024-8. URL: http://www.sciencedirect.com/science/article/ pii/0890695594900248

[7] S. Wojciechowski, P. Twardowski and M. Wieczorowski. "Surface texture analysis after ball end milling with various surface inclination of hardened steel". Metrology and Measurement Systems. Vol. XXI N ${ }^{\circ}$, pp. 145-156. 2014. DOI: $10.2478 / \mathrm{mms}-$ 2014-0014. URL: http://www.metrology. pg.gda.pl/full/2014/M\&MS_2014_145.pdf

[8] M.L. Polli. "Análise da estabilidade dinâmica do processo de fresamento a altas velocidades de corte". Tesis para optar por el grado de doctor. Faculdade de Engenharia Mecânica. UFSC Florianópolis, Santa Catarina. Brasil. 2005.
[9] A.I.S. Antonialli, A.E. Diniz and R. Pederiba. "Vibration analysis of cutting force in titanium alloy milling". International Journal of Machine Tools and Manufacture. Vol. $50 \mathrm{~N}^{\mathrm{o}} 1$, pp. 65-73. 2010. URL: https://www.researchgate.net/ publication/223092790_Vibration_analysis_ of_cutting_force_in_titanium_alloy_milling

[10] G.L. Nicola, R.P. Zeilmann e F.P. Missel. "Textura superficial no acabamento do aço endurecido em planos inclinados". Máquinas e Metais. Aranda Editora. Ano XLV, número 516. São Paulo, Brasil. 2009.

[11] M. Cosma. "Experimental studies on influence of tool path in 3-axes B.N.E.M. on inclined surfaces at 45 degrees". Academic Journal of Manufacturing Engineering. Vol. $9 \mathrm{~N}^{\circ} 4$, pp. 30. 2011. URL: http://www.eng.upt.ro/ auif/Lucrari_PDF_2011_4/30.Articol\%20 AJME_Cosma\%20Marius.pdf

[12] C.K. Toh. "Surface topography analysis in high speed finish milling inclined hardened steel". Precision Engineering. Vol. 28 $\mathrm{N}^{\circ}$ 4, pp. 386-898. 2004. DOI: $10.1016 / \mathrm{j}$. precisioneng.2004.01.001. URL: http:// www.sciencedirect. com/science/article/pii/ S0141635904000066

[13] M. Cosma. "Horizontal path strategy for 3d-cad analysis of chip area in 3-axes ball nose end milling". $7^{\text {th }}$ International Multidisciplinary Conference. Baia Mare, Romania. 2007.

[14] M. Cosma. "Geometric method of undeformed chip study in ball nose end milling". The International Conference of the Carpathian Euro-Region Specialists in Industrial Systems, $6^{\text {th }}$ edition. Baia Mare, Romania. 2006.

[15] A.E. Diniz, F.C. Marcondes e N.L. Coppini. "Tecnologia da usinagem dos metais". $7^{\text {ma }}$ ed. Artileber Editora Ltda. São Paulo, Brasil. 2010. ISBN: 85-87296-01-9.

[16] B.J. Pires. "Estudo das forças, vibrações e desgaste das ferramentas no fresamento da liga de titânio Ti-6Al-4V". Tesis para optar al grado de máster. Faculdade de Engenharia Mecânica. UNICAMP. Campinas, São Paulo, Brasil. 2011.

[17] A.J. de Oliveira. "Análise do desgaste de ferramentas no ferramentas no fresamento com Alta Velocidade de aços endurecidos". Tesis para optar al grado de doctor. Faculdade de Engenharia Mecânica. UNICAMP. Campinas, São Paulo, Brasil. 2007. 